\title{
Localization using iterative angle of arrival method sharing snapshots of coherent subarrays
}

\author{
Shun Kawakami* and Tomoaki Ohtsuki
}

\begin{abstract}
In this paper, we propose a localization method using iterative angle of arrival (AOA) method sharing snapshots of coherent subarrays. The conventional AOA method is restricted in some applications because array antenna used for receivers requires many antennas to improve localization accuracy. The proposed method improves localization accuracy without increasing elements of antenna arrays, and thus the lower costs and smaller devices are expected. First, we estimate rough location of source with each subarray-small number of antennas-in initial estimation. Then, we configurate virtual arrays by sharing snapshots based on the initial AOAs, estimate again with virtual arrays-large number of antennas-in update estimation, and update the location iteratively. Simulation results show that the localization accuracy of the proposed method is better than that of the conventional method using the same number of antennas if the appropriate virtual arrays are configurated and the phase synchronization error between two subarrays is smaller than 0.14 of a wavelength.
\end{abstract}

Keywords: localization, angle of arrival, antenna array, virtual array

\section{Introduction}

Localization of sources is attracting a great deal of interest in mobile communications and other many applications. Global positioning system (GPS) is used in various applications, such as location information service of cellular phone and car navigation system. However, nodes require to equip with exclusive receivers that are expensive. More importantly, GPS is unavailable indoor or underground. Accurate indoor localization plays an important role in home safety, public services, and other commercial or military applications [1]. In commercial applications, there is an increasing demand of indoor localization systems for tracking persons with special needs, such as elders and children, who may be away from visual supervision. Other applications need the solutions to trace mobile devices in sensor networks. Therefore, various localization techniques alternative to GPS have been researched. They are classified to two categories: lateration using distance information by more than two receivers and angulation using direction information by more than one.

\footnotetext{
* Correspondence: kawakami@ohtsuki.ics.keio.ac.jp

Graduate School of Science and Technology, Keio University, Yokohama, Japan
}

\section{Springer}

(c) 2011 Kawakami and Ohtsuki; licensee Springer. This is an Open Access article distributed under the terms of the Creative Commons Attribution License (http://creativecommons.org/licenses/by/2.0), which permits unrestricted use, distribution, and reproduction in any medium, provided the original work is properly cited.
Time difference of arrival (TDOA) method estimates the distance from propagation times through different receivers [2]. Received signal strength (RSS) method uses the knowledge of the transmitter power, the path loss model, and the power of the received signal to determine the distance of the receiver from the transmitter [3]. For lateration, a node estimates the distances from three or more beacons to compute its location. Angle of arrival (AOA) method uses array antenna to estimate direction of arrival and at least two receivers, called subarray, are required to localize sources [4]. Localization accuracy of this method is higher than that of TDOA and RSS in theory, but it is restricted in some applications, because array antenna used in receivers is large. The accuracy of AOA depends on the number of antennas, thus it requires more antennas to improve the accuracy.

Some schemes are proposed to solve the problems as mentioned above. Cooperative AOA uses only one set of acoustic modules and radio transceiver for each, if meet with certain conditions (e.g. distances between each other within a certain range) [5]. However, this scheme previously requires the distances obtained by TDOA or RSS, and its localization performance is low if the errors of the distances are large. 
In this paper, we propose an iterative localization method based on AOA. This method requires at least two subarrays each configurated of some antennas like the general AOA method. The objective of the proposed method is to improve localization accuracy without increasing antennas. First, we estimate rough location of source with each subarray-small number of antennas-in initial estimation. Then, we configurate virtual arrays by sharing snapshots based on initial AOAs, estimate again with virtual arrays-large number of antennas-in update estimation, and update the location iteratively.

Simulation results show that the performance of localization accuracy of the proposed method is better than that of conventional method using the same number of antennas if the appropriate virtual arrays are configurated and the phase synchronization error between two subarrays is smaller than 0.14 of a wavelength. The localization accuracy of the proposed method is almost identical to that of conventional method using the large number of antennas.

\section{Related works}

\section{General localization method using AOA}

AOA method uses array antenna to estimate direction of arrival and more than two subarrays are required to localize sources. Assume that there is a sufficient distance between sources and each subarray, called far field model, formulated by $r \geq 2 D^{2} / \lambda$ [6], where $r$ is a distance between source and subarray, $D$ is array aperture, and $\lambda$ is wavelength.

We consider that there are two subarrays and one source in the field. Each subarray estimates signal directions $\hat{\theta}_{1}, \hat{\theta}_{2}$. Let $\left(x_{k}, y_{k}\right)$ be the phase center location of subarray $k$ and $(\hat{x}, \hat{y})$ be the estimated location of source, then two lines are respectively written by,

$$
\begin{aligned}
& \hat{y}-y_{1}=\left(\hat{x}-x_{1}\right) \tan \hat{\theta}_{1}, \\
& \hat{y}-y_{2}=\left(\hat{x}-x_{2}\right) \tan \hat{\theta}_{2} .
\end{aligned}
$$

From Equations 1 and 2, $(\hat{x}, \hat{y})$ can be solved as

$$
\left\{\begin{array}{l}
\hat{x}=\frac{x_{1} \tan \hat{\theta}_{1}-x_{2} \tan \hat{\theta}_{2}+y_{2}-y_{1}}{\tan \hat{\theta}_{1}-\tan \hat{\theta}_{2}} \\
\hat{y}=\frac{\left(x_{1}-x_{2}\right) \tan \hat{\theta}_{1} \tan \theta_{2}+y_{2} \tan \hat{\theta}_{1}-\tan \hat{\theta}_{2}}{\tan \hat{\theta}_{1}-y_{1} \tan \hat{\theta}_{2}} .
\end{array}\right.
$$

Two non-parallel lines are sufficient to locate a position on a plane. How accurate the position is depends on the estimation accuracies of $\hat{\theta}_{1}$ and $\hat{\theta}_{2}$. With more than three subarrays, multiple intersection points are available, and one point is selected by some methods [4], for example, mean aggregation. AOA is estimated by MUSIC [7], ESPRIT [8], and so on. In this paper, we choose MUSIC for its simplicity.

\section{Array model for separated subarrays}

In [9], the environment that the AOA of a single signal impinges on two subarrays is considered. If two subarrays are assumed ideal and identical, each geometry is uniform linear array (ULA), configurated of $M$ elements and interelements spacing is $d$, steering vectors are written as

$$
\begin{aligned}
\mathbf{a}_{1}(\theta) & =\mathbf{a}_{2}(\theta) \\
& =\left[1, e^{j(2 \pi / \lambda) d \sin \theta}, \ldots, e^{j(2 \pi / \lambda)(M-1) d \sin \theta}\right]^{\mathrm{T}},
\end{aligned}
$$

where $[\cdot] \mathrm{T}$ represents the transpose operation.

Then, a steering vector for the whole array is given by

$$
\mathbf{a}(\theta)=\left[\begin{array}{rr}
\mathbf{a}_{1}(\theta) \\
e^{j}(2 \pi / \lambda) R \sin \theta & \mathbf{a}_{2}(\theta)
\end{array}\right]
$$

where $R$ is a distance between the two subarrays.

\section{The virtual array technique}

The virtual, or interpolated, array technique is researched in order to estimate the AOAs of coherent sources [10] and reduce the elements of array [11]. In this technique, the real array manifold is linearly transformed onto a preliminary specified virtual array manifold over a given angular sector. That is, an interpolation matrix $\mathbf{B}$ is designed to satisfy

$$
\overline{\mathbf{a}}(\theta)=\mathbf{B}^{H} \mathbf{a}(\theta),
$$

where $\mathbf{a}(\theta)$ and $\overline{\mathbf{a}}(\theta)$ are the steering vectors of the real and virtual array, respectively, and $[\cdot]^{\mathrm{H}}$ represents the Hermitian transpose operation. However, this technique requires to divide the field of array into some sectors and compute the interpolation matrix $\mathbf{B}$, preliminary.

\section{Proposed method}

We propose a new localization method sharing snapshots of coherent subarrays and estimating AOA iteratively. This method estimates the source location roughly in initial estimation and updates that iteratively in update estimation. The objective of the proposed method is to improve the localization accuracy without increasing elements of antenna arrays. In this section, we present the proposed algorithm based on AOA.

\section{Assumption}

Let us consider that there are two ULA subarrays and virtual arrays in the field as Figure 1. Each virtual array is configurated of self-subarray elements and other-subarray elements. We denote virtual array by VA after this. The array snapshots of each subarray configurated 


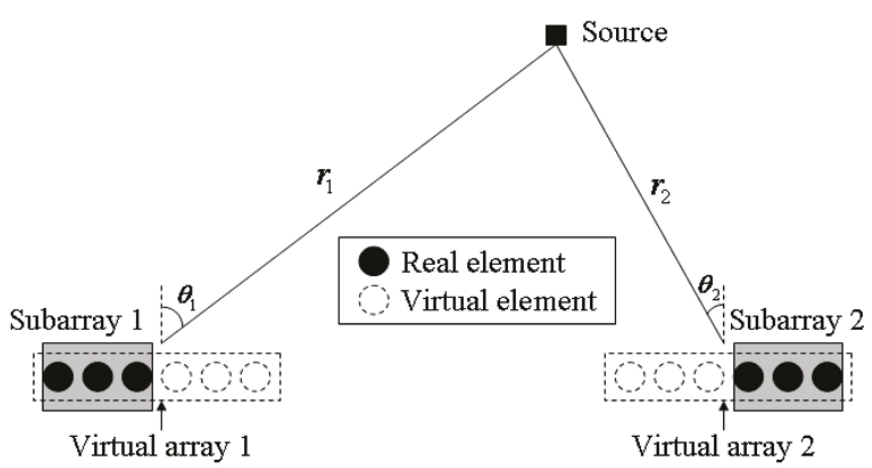

Figure 1 Proposed AOA method.

of $M$ elements at time $t$ can be modeled as

$$
\begin{aligned}
& \mathbf{x}_{1}(t)=\mathbf{a}_{1}(\theta) s(t)+\mathbf{n}_{1}(t), \\
& \mathbf{x}_{2}(t)=\mathbf{a}_{2}(\theta) s(t)+\mathbf{n}_{2}(t),
\end{aligned}
$$

where $\mathbf{x}_{k}(t), \mathbf{a}_{k}(\theta), \mathbf{n}_{k}(t)$ are the snapshots, steering vector, white sensor noise of subarray $k$, and $s(t)$ is the complex amplitude of the source, respectively.

Like Equation 5, when the reference point of each subarray is source location, array response in VA $k$ can be written as

$$
v_{m}^{k}(\theta)=a_{m}^{k}(\theta) b_{k}
$$

where

$$
\begin{aligned}
& a_{m}^{k}(\theta)=\frac{1}{r_{k}} e^{j(2 \pi / \lambda)(m-1) d \sin \theta}, \\
& b_{k}=e^{j(2 \pi / \lambda) r_{k}},
\end{aligned}
$$

$\left(1 / r_{k}\right)$ is inverse of the distance between a source and subarray $k$ that means signal fading coefficient. Note that $a_{m}^{k}(\theta)$ corresponds to array response in VA $k$ and $b_{k}$ corresponds to phase shift from a source to VA $k$.

Cooperative systems, such as virtual multiple-input multiple-output and distributed array antennas achieve high performance for capacity or location accuracy by sharing received signals, but need symbol synchronization among receivers $[12,13]$. Symbol synchronization can be achieved by transmitting pilot symbols. However, this is an unnecessary waste of bandwidth; particularly, in broadcast systems. Symbol synchronization problem is often featured in orthogonal frequency division multiplexing system, and various schemes have been proposed [14-16]. The proposed method is a kind of cooperative system and then requires the symbol synchronization. The source and each receiver is also line of sight.

\section{Initial estimation}

First, each subarray uses own correlation matrix to estimate AOA given by

$$
\begin{aligned}
& \hat{\mathbf{R}}_{1}=\frac{1}{N} \sum_{t=1}^{N} \mathbf{x}_{1}(t) \mathbf{x}_{1}^{H}(t), \\
& \hat{\mathbf{R}}_{2}=\frac{1}{N} \sum_{t=1}^{N} \mathbf{x}_{2}(t) \mathbf{x}_{2}^{H}(t) .
\end{aligned}
$$

Directions $\hat{\theta}_{1}^{(1)}, \hat{\theta}_{2}^{(1)}$ are obtained by MUSIC as follows, individually.

When the received correlation matrix is $\mathbf{R}$, the eigendeconfiguration of $\mathbf{R}$ is computed as

$$
\mathbf{R}=\mathbf{E}_{S} \boldsymbol{\Lambda}_{S} \mathbf{E}_{S}^{H}+\mathbf{E}_{N} \boldsymbol{\Lambda}_{N} \mathbf{E}_{N^{\prime}}^{H}
$$

where $\Lambda_{S}$ and $\Lambda_{N}$ are the diagonal matrices that contain the signal- and noise-subspace eigenvalues of $\mathbf{R}$, respectively, whereas $\mathbf{E}_{S}$ and $\mathbf{E}_{N}$ are the corresponding orthonormal matrices of signal- and noise-subspace eigenvectors of $\mathbf{R}$, respectively. Once the noise-subspace is obtained, the directions can be estimated by searching for peaks in the MUSIC spectrum given by

$$
P_{\text {MUSIC }}(\theta)=\frac{\mathbf{a}^{H}(\theta) \mathbf{a}(\theta)}{\mathbf{a}^{H}(\theta) \mathbf{E}_{N} \mathbf{E}_{N}^{H} \mathbf{a}(\theta)} .
$$

Then, source location is computed as Equation 3. This is the initial estimation.

\section{Update estimation}

We have, now, rough directions and distances by computing from estimated source location and known each subarray location. Next, we share the array snapshots and synchronize those as

$$
\mathbf{x}_{v 1}(t)=\left[\begin{array}{c}
\mathbf{x}_{1}(t) \\
\mathbf{x}_{2}(t) * \delta_{1}
\end{array}\right], \mathbf{x}_{v 2}(t)=\left[\begin{array}{c}
\mathbf{x}_{1}(t) * \delta_{2} \\
\mathbf{x}_{2}(t) .
\end{array}\right]
$$


$\delta_{1}, \delta_{2}$ are phase corrective functions as follows

$$
\begin{aligned}
& \delta_{1}=e^{j(2 \pi / \lambda)\left\{\left(\hat{\mathbf{r}}_{1}-\hat{\mathbf{r}}_{2}\right)+M d \sin \hat{\theta}_{2}\right\}}, \\
& \delta_{2}=e^{j(2 \pi / \lambda)\left\{\left(\hat{\mathbf{r}}_{2}-\hat{\mathbf{r}}_{1}\right)+M d \sin \hat{\theta}_{1}\right\}},
\end{aligned}
$$

where $\hat{r}_{1}, \hat{r}_{2}$ are distances and $\hat{\theta}_{1}, \hat{\theta}_{2}$ are directions estimated by subarrays 1 and 2 , respectively.

This means that the dimension of each subarray snapshots increases from $M \times 1$ to $2 M \times 1$. Each subarray uses extended correlation matrix to estimate AOA.

In case of subarray 1 , a new AOA is estimated by the virtual correlation matrix

$$
\hat{\mathbf{R}}_{v 1}=\frac{1}{N} \sum_{t=1}^{N} \mathbf{x}_{v 1}(t) \mathbf{x}_{v 1}^{H}(t)
$$

and the array response of VA 1 in the $n$th iteration is given by

$$
v_{1, m}^{n}(\theta)=\left\{\begin{array}{cc}
\frac{1}{\hat{r}_{1}} e^{j(2 \pi / \lambda)(m-1) d \sin \theta} & (1 \leq m \leq M) \\
\frac{1}{\hat{r}_{2}} e^{j(2 \pi / \lambda)(m-1) d \sin \hat{\theta}_{2}^{(n-1)}} & ((M+1) \leq m \leq 2 M)
\end{array}\right.
$$

Note that $\theta$ is the variable and $\hat{\theta}_{2}^{(n-1)}$ is the constant estimated in previous iteration. This virtual steering vector does not need the interpolation matrix as Equation 6. Assume that $\hat{\mathbf{U}}_{N 1}$ is the noise-subspace of $\hat{\mathbf{R}}_{v 1}$ and $\mathbf{v}_{1}^{n}(\theta)=\left[v_{1,1}^{n}(\theta), v_{1,2}^{n}(\theta), \ldots, v_{1,2 M}^{n}(\theta)\right]$ is the steering vector, MUSIC spectrum in VA 1 is given by

$$
P_{\text {MUSIC }}^{1}(\theta)=\frac{\mathbf{v}_{1}^{n}(\theta)^{H} \mathbf{v}_{1}^{n}(\theta)}{\mathbf{v}_{1}^{n}(\theta)^{H} \hat{\mathbf{U}}_{N 1} \hat{\mathbf{U}}_{N 1}^{H} \mathbf{v}_{1}^{n}(\theta)} .
$$

Similary, MUSIC spectrum in VA 2 whose steering vector is $\mathbf{v}_{2}^{n}(\theta)$, is given by

$$
P_{\text {MUSIC }}^{2}(\theta)=\frac{\mathbf{v}_{2}^{n}(\theta)^{H} \mathbf{v}_{2}^{n}(\theta)}{\mathbf{v}_{2}^{n}(\theta)^{H} \hat{\mathbf{U}}_{N 2} \hat{\mathbf{U}}_{N 2}^{H} \mathbf{v}_{2}^{n}(\theta)} .
$$

From Equations 21 and 22 we get new directions $\hat{\theta}_{1}^{(n)}$ and $\hat{\theta}_{2}^{(n)}$ in the $n$th iteration, and thus estimate the new source location. The proposed method iteratively updates the estimates of the directions and source locations.

\section{Virtual array configuration}

We can consider four methods about virtual array configuration as shown in Figure 2 for two subarrays. Each virtual array has the different steering vector because the elements have different order. In Figure 2, the reference point means the phase reference for each element of array antenna. The steering vector includes the

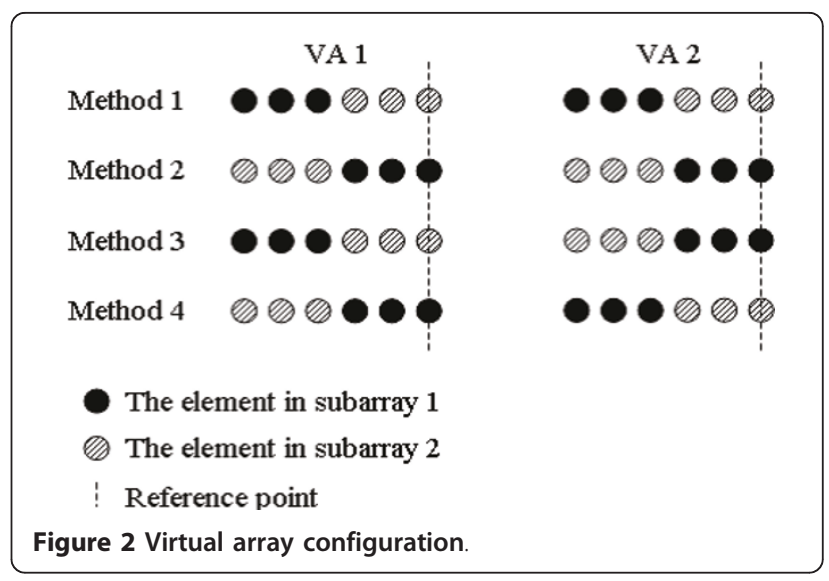

distance between the reference point and each element of array antenna. Then, the reference point is needed to compute the distance to compose the steering vector.

Figure 3 shows root mean square errors (RMSEs) comparison of four methods. Assume that the positions of subarrays 1 and $2,(M=4)$, are $(0,0),(100 \lambda, 0)$, and a source is at $(50 \lambda, 50 \lambda)$. From Figure 3, localization accuracy is high when a reference point of virtual array is a real element. This is because elements of steering vector of virtual array correspond to elements of virtual correlation matrix. Method 4 indicates the best performance because both VA 1 and VA 2 in method 4 use the real element, the element of self-subarray, as the reference point. Note that, VA 2 in method 1 and VA 1 in method 2 also use the real element as the reference point. Figure 3 also indicates the iteration count $n=5$ of method 4 is enough to improve the localization accuracy.

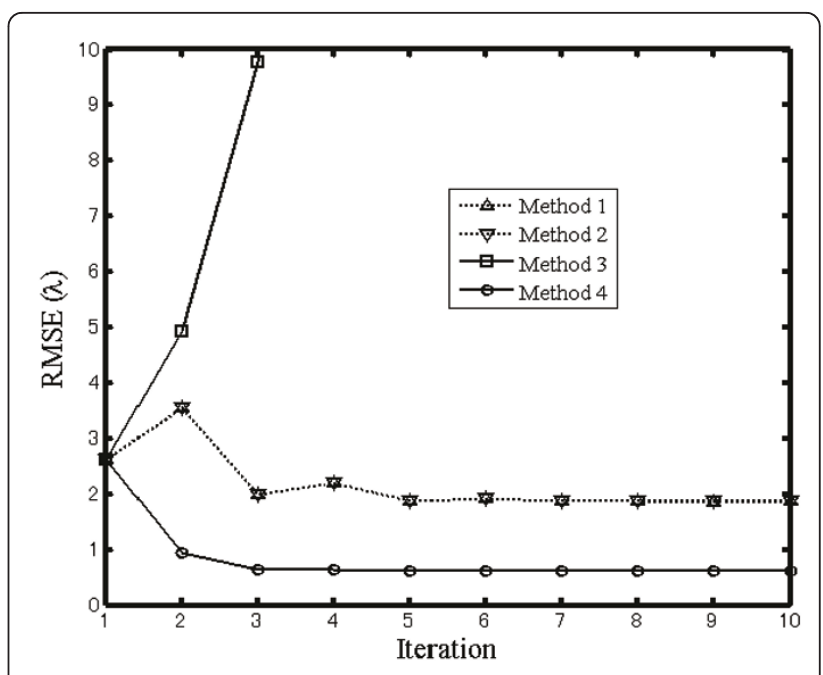

Figure 3 RMSE comparison of four methods 
Table 1 Simulation parameters

\begin{tabular}{cc}
\hline Number of sources & 1 \\
\hline Geometry of subarray & ULA \\
\hline Interelement spacing & $\lambda / 2$ \\
\hline Number of snapshots & 128 \\
\hline Noise model & AWGN \\
\hline AOA estimation method & MUSIC \\
\hline Spectral resolution & $0.1^{\circ}$ \\
\hline Simulation runs & 10000 \\
\hline
\end{tabular}

When virtual array configuration is based on method 4 , steering vectors of VA 1 and VA 2 in the $n$th iteration can be represented, respectively, as

$$
\begin{gathered}
v_{1, m}^{n}(\theta)=\left\{\begin{array}{cc}
\frac{1}{\hat{r}_{2}} e^{j(2 \pi / \lambda)(m-1) d \sin \hat{\theta}_{2}^{(n-1)}} & (1 \leq m \leq 3) \\
\frac{1}{\hat{r}_{1}} e^{j(2 \pi / \lambda)(m-1) d \sin \theta} & (4 \leq m \leq 6)
\end{array}\right. \\
v_{2, m}^{n}(\theta)=\left\{\begin{array}{cc}
\frac{1}{\hat{r}_{1}} e^{j(2 \pi / \lambda)(m-1) d \sin \hat{\theta}_{1}^{(n-1)}} & (1 \leq m \leq 3) \\
\frac{1}{\hat{r}_{2}} e^{j(2 \pi / \lambda)(m-1) d \sin \theta} & (4 \leq m \leq 6)
\end{array}\right.
\end{gathered}
$$

\section{Simulation results}

In this section, we examine the localization performance of our proposed method. We use common simulation parameters over all simulations as Table 1. The location of a source and each subarray is as Figure 4. A source is generated in random to show the proposed method does not depend on the source location.

First, the phase synchronization between two subarrays is assumed as perfect. In other words, $\delta_{1}, \delta_{2}$ in

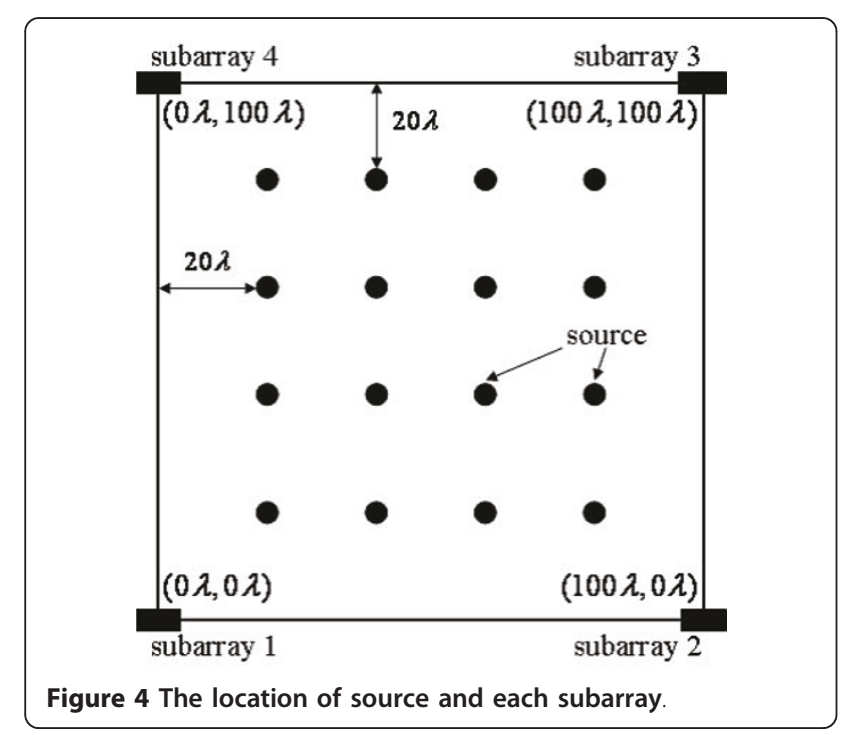

Equations 17 and 18 are exact. We compare the proposed method to three conventional methods. Conv. ( $M$ $\times K$ ) means the conventional method that uses $K$ subarrays each configurated of $M$ elements.

Prop. is the proposed method that uses two subarrays each configurated of three elements, the virtual array configuration of Prop. is based on method 4, and the iteration count $n=5$. The purpose of our proposed method is to improve the localization accuracy without increasing the number of antennas.

In Figure 5, the RMSEs of the location estimates for all the methods versus signal-to-noise ratio (SNR) are shown. Prop. performs asymptotically close to Conv. (6 $\times 2)$ and Conv. $(6 \times 4)$, and outperforms Conv. $(3 \times 2)$. This is because Prop. can use more snapshots than Conv. $(3 \times 2)$. Prop. shows the more robustness, particularly in low SNR. We stress that Conv. $(6 \times 2)$ and Conv. $(6 \times 4)$ use more antennas than Prop.

In Figure 6, the cumulative distribution function (CDFs) of location RMSEs at SNR $=0 \mathrm{~dB}$ versus the error distance, $0.5 \lambda$ intervals, are shown. The probability of Prop. in the small errors, less than $1 \lambda$, is higher than that of Conv. $(6 \times 2)$, whereas in the large errors, is also higher. In Prop., AOA is estimated using the parameters (directions and distances) estimated in the previous iteration. Thus, the estimation errors in the $(n-1)$ th iteration are larger, the localization accuracy of Prop. in the $n$th iteration is also larger.

Figures 7 and 8 show the MUSIC spectrum of the conventional method $(n=1)$ and the proposed method $(n=5)$. The maximum spectrum of the proposed method is closer to true AOA than that of the conventional method. At the same time, MUSIC spectra of the

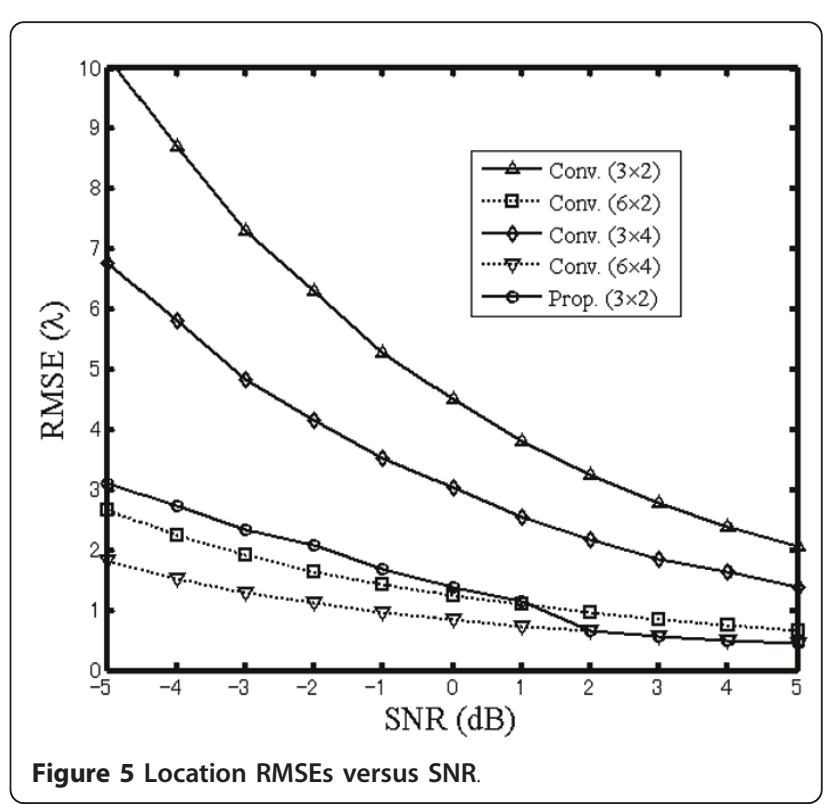




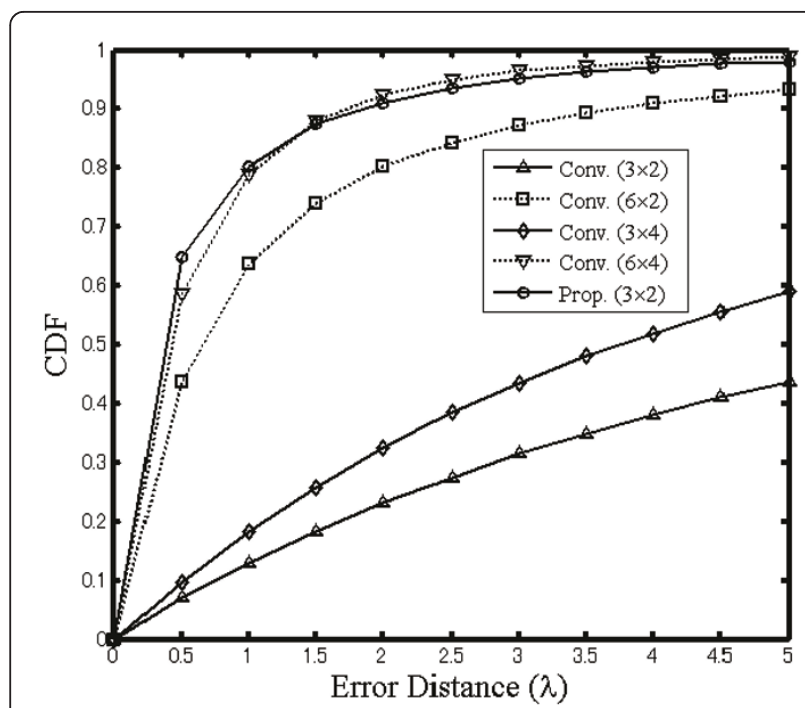

Figure 6 CDFs of location RMSEs versus the error distance.

proposed method have spurious peaks because the proposed method in update estimation uses the snapshots of the other subarray. However, these spurious peaks are much lower than the maximum spectra, true peaks, then we can distinguish these peaks.

Next, we evaluate the effect of the phase synchronization error between two subarrays. Note that the phase synchronization error is defined as the error arising among different separated receivers. We assume that two subarrays are located in the different far field, then those are not connected by cable cannot be synchronized perfectly. Figure 9 shows location RMSE of the proposed method versus the synchronization error between two subarrays, where method 4 is used for

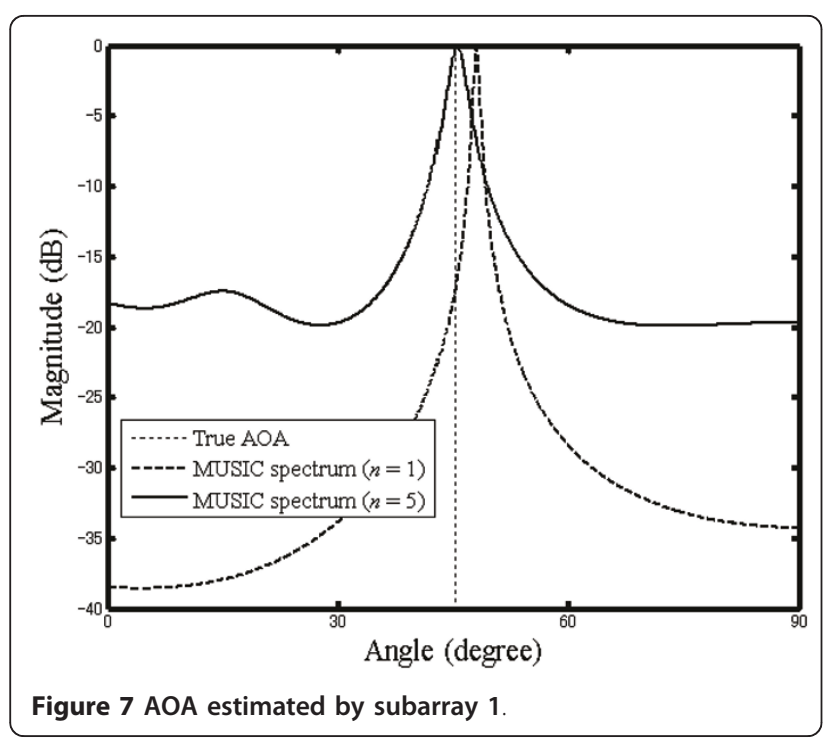

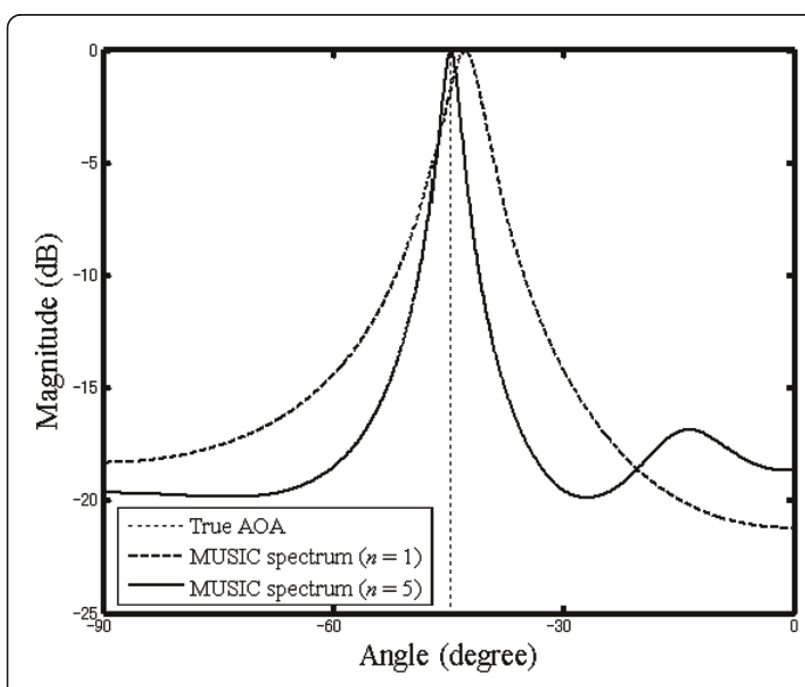

Figure 8 AOA estimated by subarray 2 .

virtual array configuration and iteration time is 5 . The synchronization error is added to $\delta_{1}, \delta_{2}$, and its variance is defined as Gaussian distribution.

We can see that phase synchronization between two subarrays is important for the proposed method because RMSE becomes larger as error variance increases. The proposed method can achieve smaller RMSE the conventional one when the error variance is smaller than $0.02 \lambda^{2}$.

\section{Conclusion}

In this paper, we proposed a new localization method based on AOA. The objective of the proposed method is to improve localization accuracy without increasing

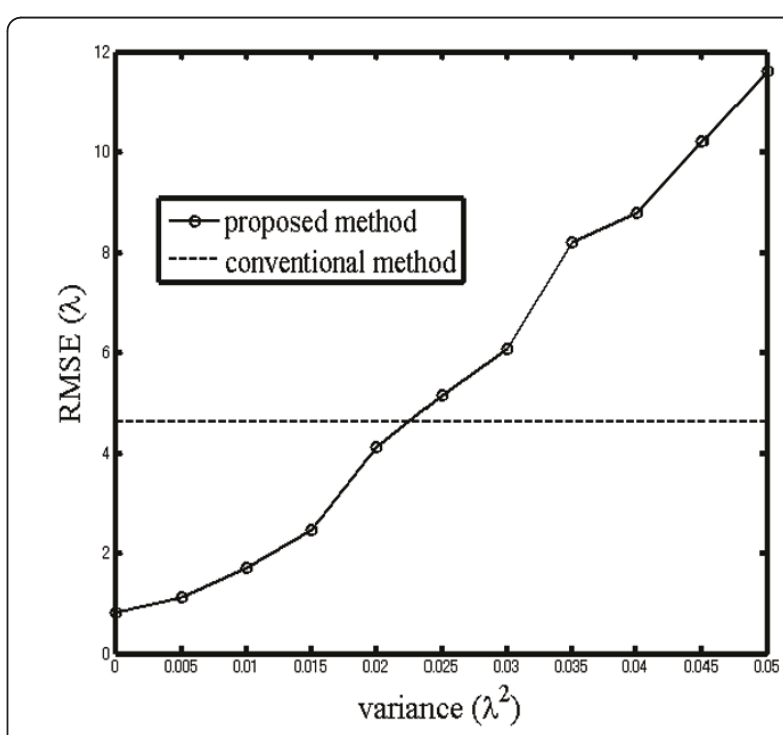

Figure 9 Location RMSEs versus phase synchronization error. 
antennas. This method estimates rough source location by initial estimation, share snapshots of coherent subarrays, and iteratively update source location by update estimation. We showed that the proposed method localizes a source more accurately than the conventional method when the reference point of virtual array is a real element and the phase synchronization error between two subarrays is smaller than 0.14 of a wavelength.

\section{Abbreviations \\ AOA: angle of arrival; CDFs: cumulative distribution function; GPS: global positioning system; RMSEs: root mean square errors; RSS: received signal strength; TDOA: time difference of arrival; ULA: uniform linear array.}

\section{Acknowledgements}

This work was supported by Ohtsuki Laboratory, the Department of Computer and Information Science, Keio University. Part of this paper was presented at the Asia-Pacific Signal and Information Processing Asso- ciation (APSIPA ASC 2009) and at the IEEE International Conference on Wireless Information Technology and Systems (ICWITS 2010).

\section{Competing interests}

The authors declare that they have no competing interests.

Received: 14 November 2010 Accepted: 24 August 2011

Published: 24 August 2011

\section{References}

1. K Pahlavan, P Krishnamurthy, J Beneat, Wideband radio channel modeling for indoor geolocation applications. IEEE Commun Mag. 36(4), 60-65 (1998). doi:10.1109/35.667414

2. $\quad \mathrm{L}$ Jun, $C$ Qimei, $T$ Xiaofeng, Z Ping, A method to enhance the accuracy of location systems based on TOA-location algorithms. in ITS Telec Proc Conference, 979-982 (2006)

3. F Reichenbach, D Timmermann, Indoor localization with low complexity in wireless sensor networks. IEEE Ind Info, 1018-1023 (2006)

4. S Zhilong, PYT Shing, Precise localization with smart antennas in Ad-Hoc networks, in IEEE Globecom Conference, pp. 1053-1057 (2007)

5. THui, W Shuang, X Huaiyao, Localization using cooperative AOA approach. Wicom Networking and Mobile Computing, 2416-2419 (2007)

6. S Kuldip, Antenna near field intensity predection, in Proceedings of the International conference on Electromagnetic Interference and Compatibility, pp. 109-114 (1999)

7. H Krim, M Viberg, Two decades of array signal processing research. IEEE Signal Process Mag. 13(4), 67-94 (1996). doi:10.1109/79.526899

8. B Friedlander, AJ Weiss, Direction finding using spatial smoothing with interpolated arrays. IEEE Trans Aerosp Electron Syst. 28, 574-587 (1992). doi:10.1109/7.144583

9. F Athley, C Engdahl, Direction-of-arrival estimation using separated subarrays, in Proceedings 34th Asilomar Conference Signals, Systems, Computation 1, 585-589 (2000)

10. B Friedlander, Direction finding using an interpolated array. IEEE Signal Process Lett. 5, 2951-2954 (1990)

11. AB Gershman, JF Bohme, A note on most favorable array geometries for DOA estimation and array interpolation. IEEE Signal Process Lett. 4, 232-235 (1997). doi:10.1109/97.611287

12. M Seo, M Rodwell, M Madhow, A feedback-based distributed phased array technique and its application to $60-\mathrm{GHz}$ wireless sensor network, in IEEE MTT-S International Microwave Symposium Digest, pp. 683-686 (2008)

13. Y Hua, Q Zhang, Z Niu, A cooperative MAC protocol with virtual-antenna array support in a multi-AP WLAN system. IEEE Trans Wirel Commun. 8(9), 4806-4814 (2009)

14. J Beek, M Sandell, PO Borjesson, ML estimation of time and frequency offset in OFDM systems. IEEE Trans Signal Process. 45, 1800-1805 (1997) doi:10.1109/78.599949
15. D Lee, $K$ Cheun, A new symbol timing recovery algorithm for OFDM systems. IEEE Trans Consumer Electron. 43, 767-775 (1997). doi:10.1109/ 30.628706

16. A Armada, M Ramon, Rapid prototyping of a test modem for terrestrial broadcasting of digital television. IEEE Trans Consumer Electron. 43, 1100-1109 (1997). doi:10.1109/30.642377

\section{doi:10.1186/1687-6180-2011-46}

Cite this article as: Kawakami and Ohtsuki: Localization using iterative angle of arrival method sharing snapshots of coherent subarrays. EURASIP Journal on Advances in Signal Processing 2011 2011:46.

\section{Submit your manuscript to a SpringerOpen ${ }^{\mathcal{O}}$ journal and benefit from:}

- Convenient online submission

- Rigorous peer review

- Immediate publication on acceptance

- Open access: articles freely available online

- High visibility within the field

- Retaining the copyright to your article

Submit your next manuscript at $>$ springeropen.com 